\title{
A Survey of Unconstrained Face Recognition Algorithm and Its Applications
}

\author{
Ranbeer Tyagi ${ }^{1}$, Geetam Singh Tomar ${ }^{2}$ and Namkyun Baik ${ }^{3 *}$ \\ ${ }^{1}$ Research Scholar, Deptt. of Electronics \& Communication UTU, Dehradun \\ (Uttarakhand), India- 248007 \\ ${ }^{2}$ Deptt. of Electronics \& Communication \\ THDC -IHET, Tehri Garhwal Uttarakhand, India-249124 \\ ${ }^{3}$ Korea Internet \& Security Agency \\ Jungdaero 135, Songpa, Seoul, Korea-135950 \\ ${ }^{1}$ ranbeertyagi85@gmail.com, ${ }^{2}$ gstomar@ieee.org, ${ }^{3}$ white-knight@naver.com
}

\begin{abstract}
Face-recognition is becoming common among the section of study in computer-vision, while it is also one of the very effective programs of comprehension and image-analysis. It may be employed for both ID and confirmation. At the moment, there are lots of means of front watch face-recognition. Nicely when just one instant picture per course can be obtained nevertheless, a handful of them can perhaps work. In this paper, we discuss the different face recognition techniques and find a better method for pose variation, nonuniform motion blur and Illumination by using a Reference face graph for face recognition. One example image' problem and two generalized eigenface algorithms are proposed. Face-recognition has been analyzed thoroughly; nevertheless, real world facerecognition stays a job that is difficult. The interest in unconstrained useful facerecognition is increasing using the surge of online media, for example, video-surveillance video, and internet sites wherever encounter evaluation is of substantial significance. Face-recognition is approached by us within data theory's framework. We identify an unfamiliar encounter utilizing an exterior Reference Face Graph (RFG). There is an RFG produced by evaluating it towards the encounters within the built RFG and acknowledgement of the given encounter is attained. Centrality steps are used to recognize encounters that were unique within the Reference Face Graph.
\end{abstract}

Keywords-Face Recognition, Image Processing, Network Security and Surveillance, Eigenface, Centrality measure, Alignment-free, Pose robust

\section{Introduction}

Face-recognition is a kind of software program that may determine a particular person in an electronic picture by evaluating and examining designs. Face-recognition methods are generally employed for protective reasons, but is progressively getting used in a number of other additional programs and functions. Facial-recognition to distinguish among people is used by the movement gaming console, for instance. Facial-recognition application to greatly help automate person marking in pictures is used by Myspace. Recognition works in Myspace: every time a person is labeled in an image, the program software retailers detail the cosmetic traits that are a person's. The machine utilizes that info to recognize exactly the same encounter in various pictures, and certainly will consequently recommend marking these pictures while enough information continues to be gathered in regards to an individual to recognize them. In the networked globe of

* Corresponding Author 
today, the requirement to keep up with the protection of bodily or info harm has become equally progressively challenging and essential. From the time the pc is broken into by hackers, or safety breaches in a government or company building [1], [10], [11]. In many of those offenses, the crooks were benefiting from a simple drawback within the traditional access-control methods: the systems don't give entry by "who we're", but by "what we've", such as for instance identification cards, secrets, accounts, FLAG figures, or mom's maiden name. We are not actually defined by any of those means. Instead, they simply are means. It means without stating when somebody takes, copies, or receives these identification means, he/she will have the ability to gain access to our individual home or our information any moment they need. Lately, engineering became open to permit confirmation of "accurate" identification that was a person [12], [13]. This engineering relies on a field called "biometrics". Biometric access-control is some facets of the individual's behaviour, or automatic ways of confirming or realizing the identification of the living person about the foundation of some physical traits, for example fingerprints or cosmetic functions. They're challenging to create because biometric methods determine an individual by natural faculties. One of the numerous biometric identification techniques, the physical methods (fingerprint, encounter, genetics) are far steadier than methods in attitudinal class (keystroke, voice-print). This is because functions that are physical in many cases are seldom alterable, except by damage that is serious [14], [15]. The attitudinal designs, on the other-hand, might vary because of exhaustion tension or disease. Nevertheless, attitudinal IDs possess the benefit of being non-intrusive. Individuals are much more comfortable signing their titles or talking with a microphone than putting their eyes or providing a fall of blood sequencing [16].

Face-recognition is among the several biometric techniques that hold the merits of reduced intrusiveness and equally large precision. It's the precision without having to be invasive of the physical strategy. Because of this, because the early 70s (Kelly, 1970), face-recognition has attracted the interest of scientists in areas from protection, mindset, and image-processing, to computer-vision. Numerous calculations have now been suggested for face-recognition; for comprehensive study please observe Chellappa (1995) [2] and Zhang (1997) [3]. Although access-control and community protection were programs widely mentioned by it, face-recognition has additionally confirmed use- total in additional media information places that are processing. Whilst multimedia's surge has been seen in the previous decade, the running and evaluation of media information, including movies and pictures, are of curiosity that is wide [17], [18]. Among these information, movies and encounter pictures have a portion that is big. The acknowledgement of the encounters in contexts that are various allows numerous programs, for example social media security checking, and individual -pc conversation. For instance, from road monitoring cameras assisting the FBI to recognize the suspects [4], pictures of the suspects obtained within the current occasion of Boston race bombings.

Face-recognition contains the reports of confirming or instantly distinguishing an individual from a movie or image sequences. Encounter ID describes identifying the individual, provided like a probe's identification, from all the other applicants within the gallery. Encounter certification or encounter confirmation may be of determining whether a confirmed set of pictures are of exactly the same person. Remarkable outcomes have now been documented on standard sources and though face-recognition has been analyzed thoroughly [5], [6], [7], face-recognition that is unconstrained would be practical to use, which is nevertheless challenging because of the following elements:

Offer deviation: the face area to not become unrecognized will come under arbitrary poses.

Misalignment: Since the looks usually are detected by an automated face alarm [8], [19], the cropped looks are not aimed. Nonetheless, because stance is required by many 
attribute descriptors before element removal, imbalance degrades the performance of a face-recognition system.

Light alternative: the look of the face area would range notably under different light, and along with the lighting circumstances influence the illumination on the face.

Appearance deviation: The face images and expressions that are various may differ.

It is well known the precision of face-recognition methods drops very quickly in configurations that are unconstrained [9], [20]. This is often related to degradations present as a result of blur, modifications in lighting, and expression occlusions blur, particularly, warrants specific interest because of the ubiquity of cell phones and hand held imaging products. Coping with camera-shake is really just a related issue since it influences image quality, while tripods impede flexibility. Furthermore, in built devices like gyros for example, in realizing the camera movement and accelerometer shave their very own restrictions. Within an atmosphere that is unchecked, present and lighting may also differ, more compounding the issue. This paper's emphasis is on creating a program that may identify encounters across non uniform (space -version) cloud, and different lighting and present [20]. Using the broad implementation of face identification methods in programs from de-duplication to cellular system unlocking, protection from the face spoofing assaults demands elevated interest; such assaults could be quickly released via published pictures, movie replays, and 3D goggles of the experience. We tackle the issue of encounter spoof recognition from the printing (picture) and replay (photo or movie) assaults on the basis of the evaluation of picture distortion (e.g., area reflection, moiré design, colour distortion, and form deformation) in spoof encounter pictures (or video frames). The applying site of curiosity is smart phone uncovered, considering the fact that the expanding quantity of smart phones possess the encounter unlock and cellular repayment abilities [26].

\section{Face Recognition Techniques}

Beginning in the 1970s, face-recognition was addressed like a 2D pattern recognition difficulty [21]. The miles between important details wherever used to understand identified people, e.g., calculating the gap involving the eyes or additional significant details or measuring unique perspectives of facial factors. Nevertheless, it is necessary for the face-recognition techniques to become fully automatic. Recognizing the face is such a tough, yet nevertheless exciting dilemma that has captivated researchers with diverse skills: pattern-recognition mindset, neural systems and computer graphics. The following approaches are accustomed to face-recognition [22], [23].

1. Holistic Related Techniques

2. Function-based (architectural) Techniques

3. Hybrid Techniques

1. Holistic Related Techniques: In this system, the experience spot that is taken into consideration as feedback data in natural strategy. One of the finest illustration of healthy methods are Eigenfaces, (hottest way of face-recognition), Primary Component Evaluation, Linear Discriminant Analysis, and independent element analysis etc.

2. Function-based (architectural) Techniques: In this method,nearby capabilities such as eyes, nose, and mouth are first of all extracted and their destinations and nearby research (geometric and/or look) are fed into a structural classifier. A huge concern for characteristic removal methods is characteristic "recovery", that is once the program tries to recover functions which might be hidden as a result of big modifications, e.g. Head Present whenever we are matching' a frontal graphic [24 ] Distinguishes between three different removal practices:

i. Common practices based on sides, lines, and curves 
ii. Feature-theme-based methods

iii. Structural matching strategies that take into account geometric Limitations on the capabilities.

3. Hybrid Techniques: Hybrid face recognition programs make use of a mix of both healthy. Generally, 3D Pictures are used in hybrid techniques. The graphic of a person's encounter is captured in 3D, letting the device see perhaps the shapes of the face or temple, or the shapes for example. Since the program uses an axis of dimension, which provides it enough information to construct the full experience, and range a face in report could provide. The 3D process frequently proceeds as follows: Placement Recognition, Description, Rendering and Corresponding. Diagnosis - Recording an experience the checking an image or photographing someone's face in time that is real. Position Determining direction, dimension and the positioning of the head. Measurement Assigning sizes to each bend of the face area to create a format with particular focus on the nose's position and the surface of the eye. Representation - Changing the template in to a code - an illustration of Matching and the face - Researching the received info in the current repository with people.

\section{Face Recognition Algorithms}

Within this portion, we will identify two types that are representative. The Eigenface method applies the Karhonen-Loeve (KL) convert for feature extraction. It greatly reduces the facial characteristic aspect but retains reasonable power that is discriminating. The neural network strategy, though some alternatives of the algorithm work with function extraction as well, primarily gives a refined modeling plan for estimating likelihood densities in the pattern recognition section.

\section{Eigenface:}

As stated, one of the targets the feature removal program wants to reach will be to increase the effectiveness [22]. One simple way to accomplish that goal is using alternate ortho-normal bases other than the normal basics. One basis will be the Karhonen- Loeve (KL). The eigenvectors of the covariance matrix of the face vector Y form kL bottoms. In the high-dimensional "face" room, only the first few eigen-values have values that are huge. Within the subspace constituted from the first several eigenvectors to put it differently, energy largely locates. Consequently, an excellent pressure may be accomplished by letting these eigenvectors with huge eigen-values to symbolize the face $\mathrm{Y}$,

$$
Y=\sum_{j=1}^{K} \hat{y}_{j} v_{j}
$$

Wherever $\mathrm{v}$ will be $\mathrm{K}$ and the eigenvector is usually much smaller than original vector $\mathrm{N}$. Because encounter images are looked like by the eigenvectors from the first several eigenvalues, KL basics can also be called eigenfaces. The representation is well known in statistics literature while the main component analysis. It is optimum within efficiency's perception: for any given $\mathrm{K}<\mathrm{N}$, The illustration has the minimum mean square problem among all feasible estimates of $\mathrm{Y}$ that employs $\mathrm{K}$ orthonormal vectors. However, it generally does not mean that the rendering is ideal of discriminating power, which relies more on the separation between diverse faces rather than the spread of all faces, while in the sensation.

\section{Neural Network:}

In principle, the favorite back propagation neural network might be qualified to identify faces in pictures specifically [22]. For perhaps a picture with dimension that is 
reasonable, nonetheless, the community can be so difficult to coach and quite complicated. As an example, when the graphic is pixels, inputs of the network's number will be 16. Neural system is frequently placed on the pattern recognition phase in place of the element extraction cycle to lessen difficulty. Sung and Poggio's face detection protocol (Sung, 1995) down-samples a face image in to a 19x19 cosmetic feature vector before they apply the elliptical mean clustering to product the distributions of the "face samples" and also the "non-experience samples". An example of the neural classifier is the Probabilistic Selection- centered Neural Network (PDNN) (Lin, 1997). PDNN does not have the entirely connected topology. Instead, the circle is divided by it into $\mathrm{E}$ subnets. Each subnet is focused to identify anyone inside the repository. PDNN utilizes the Gaussian initial purpose for the nerves, and each "encounter subnet's" output will be the outputs' measured summary. To put it differently, the face subnet quotes the reality density utilizing the mix that is popular -of- model. Compared to the AWGN scheme, a sophisticated and much more adaptable product is provided by combination of Gaussian for approximating the real probability densities within the encounter area.

\section{Reference Face Graph:}

A reference face graph (RFG) [8] is a composition of nodes and also the dyadic interactions (edges) involving the nodes. A reference experience is a node addressing just one individual while in the guide encounter graph. Each research face has numerous photographs with numerous poses, words, and illumination. All-the photos of those reference faces create a set named the guide foundation set. We utilize this because we represent each probe encounter or gallery encounter as a linear mixture of the characteristics towards the reference people in the reference schedule collection the period base set. A foundation descriptor of a graphic is a vector that identifies the image when it comes to its likeness towards the reference looks. A guide face descriptor (RFD) is defined by incorporating the RFG node centrality measurements to the foundation descriptor.

\section{Normalized Pixel Variation:}

We recommend a solution to tackle difficulties in unconstrained face detection, for example haphazard offer versions and occlusions. First, a fresh photograph characteristic named Normalized Pixel Variation (NPV) is recommended [25]. NPV feature is calculated while the variation to total percentage between two values impressed from the Weber Fraction in experimental mindset. The new element bounded is level invariant, and it is able to reconstruct the initial picture. So that the discovered regulations can partition complicated face manifolds second, we propose a deep quadratic tree to master the perfect part of NPV attributes as well as their mixtures. In this manner, merely a solitary delicatestream classifier is required to handle unconstrained face-detection. Moreover, we show that the NPV attributes could be efficiently acquired from a research stand, and the recognition theme could be quickly scaled, building the proposed encounter sensor very fast. Fresh effects on three public encounter datasets (FDDB, GENKI, and CMU-MIT) demonstrate the proposed strategy achieves express-of-the-craft efficiency in detecting unconstrained people with arbitrary offer variations and occlusions in cluttered scenes.

\section{Applications of Face Recognition}

Face recognition is also not useless in-human computer connection, virtual reality, database retrieval, multimedia, computer enjoyment, information protection e.g. Biometric e.g, oS, medical records. Personal Detection - Passports licenses, Automatic identity verification - law-enforcement e.g, edge adjustments. Movie surveillances, 
investigation, Personalized Safety - home video surveillance system, driver tracking system.

Face Detection: face people are identified by recognition devices. Face recognition methods establish the clear presence of a licensed individual rather than only examining whether a logical detection (identification) or key has been used or whether the individual appreciates the secret particular identification numbers (Hooks) or accounts. Listed here are example.

Because there are situations where precisely the same individual was assigned more than one identification number, to eradicate copies in a nationwide voter registration program. The face recognition process immediately examines the face area pictures of the voters and doesn't utilize identity numbers and the others to differentiate the person. Manual assessment is required to be sure they're indeed unique folks so as to eliminate copies, when the top-two matched encounters are highly just like the query encounter picture.

Access-Control: in several including computer logon or workplace entry, of the accessibility control programs, the size of the crowd that needs to be recognized is not somewhat large. The face area pictures can also be grabbed under organic circumstances, such as Front encounters and indoor illumination. The face recognition process with this application is capable of accuracy that is superior without much cooperation from any person. Listed here is the example. Face-recognition technology is employed to continually observe who is in front of a PC terminal. It permits an individual to depart at the final without closing documents and visiting out. When the person leaves to get a predetermined period, a screensaver hides the job and disables keyboard \& the mouse. The user comes home and it is acknowledged, if, the screensaver opens because it was left and also the preceding session appears. Every other consumer who tries to logon without agreement is rejected.

Security: Today, security is really a main matter at airports as well as for flight team workplace and guests. Airport protection techniques that use face-recognition technology have now been applied at several airports around the world. Listed below are the 2 instances.

In April 2001, Fresno Yosemite Overseas (FYI) airport in Florida implemented Viisage's face recognition technology for airport security reasons. The machine is designed to alert FYl's airport public safety officers when a person matching the looks of a suspect that was known enters the airport's safety checkpoint. Anyone acquiesced by the system could have investigative operations by public safety officials. Computer security has also noticed the effective use of face-recognition technology. To avoid somebody else from changing files or transacting with others once the approved individual leaves the pc terminal for a short-time, customers are repeatedly authenticated; examining the person before the monitor or in person could be the same certified individual who logged in.

Graphic database inspections: Seeking impression listings of immigrants, gain individuals qualified motorists and authorities reservations.

Standard ID verification: Electoral enrollment, bank, electronic-commerce, distinguishing babies Passports, Employee IDs.

Surveillance: Like stability purposes in public spots, surveillance by face-recognition techniques includes a low user satisfaction stage, or even lower. Other divisors experience orientations along with free illumination circumstances create the deployment of face recognition methods for large-scale detective an activity that is complicated. Listed here 
are some cases of face-based monitoring. The city council promises engineering has assisted to attain a $34 \%$ drop in transgression since its ability. Similar devices have come into place in Manchester, England. In 1999, Visionics was given by the Institute of Justice to develop clever technology that was CCTV.

Simple people tagging: Facebook's computerized label tip feature, which used face recognition to recommend people you may need to draw within your photos, got individuals sizzling under the collar. Nevertheless, whether you will find it scary or awesome, face-recognition for people tagging undoubtedly saves time. It's currently for sale in Apple's iPhoto Picasa and on Facebook. Gaming: Picture and face-recognition is getting a complete new dimension to gaming. Microsoft's Kinect opened gambling to fresh readers by completely doing away with equipment controllers and sophisticated motion sensing features have given a whole new rental of existence to the Xbox 360 Console. Meanwhile, start-up Viewdle recently launched a casino game that uses face-recognition to choose whether you're vampire or a human, placing the point to get a combat between the two species. We're certain to see additional examples of face recognition in activities as time goes by too - with all sorts of fascinating options.

Cost evaluation: In a shop and wish to learn if you're finding a deal that is good? Just break a picture of the product you intend to purchase and programs like eBay and Google Buyer -possessed Red Laser will give you the sort of knowledge shopkeepers wished you didn't have knowledge of. Although Red Laser can be a barcode reading software, which can inform you how much something you're interested in selling is at regional merchants and online, Google Buyer punches meaning you're able to have an image of it rather. Acquisitions you check might be rescued inside your record

Producing intellectual notes: with going for a look at Deja vu for aesthetic notes of points you want to remember utilizing your mobile phone's camera, you could do. This app makes it possible to plan photographs you've taken as pointers, letting you incorporate records, tags and spot data. Image acknowledgement is created in to label many book CDs, DVDs and quickly draw and wine brands, getting a supplementary stepout of the method of getting yourself organized. Discover our more in-depth coverage below. For retrieval that is later.

Determining TV-shows: Certainly One of quite a few apps planning to be 'Shazam for TV', TV talk is definitely a software that discovers the TV show you're watching, simply by directing your camera at the monitor. Within one second, it will work out the display or ad that you will be enjoying. From there, people will have a way to share details of the exhibit viewing via Facebook or Twitter, with a review connected. The Israeli startup behind this program is allowing it to be used by promoters like a 'call to action', too. You may be seeing an advertisement for a fresh treat; by going for a photo of the monitor with TVtalk, you could be subsequently taken to a coupon entitling you to a free sample.

\section{Conclusion}

It's our view that study in face-recognition is definitely an enjoyable area for many years in the future and will preserve many researchers and designers chaotic. Face recognition can be an equally tough and essentially acknowledgement approach. Among all the biometric strategies, face recognition technique includes one wonderful benefit, which is its individual-friendliness (or low-intrusiveness). On its own applications and Face-Recognition, we have offered an introductory Literature review within this document. We've covered problems like the general platform for face-recognition, factors 
that may affect the recognizer's functionality, and lots of condition-of-the- face recognition algorithms.

\section{References}

[1] “Computer Security Threat On Rise - U.S. Survey”, Reuters News, March 7, 1999.

[2] R. Chellappa, C.L. Wilson and S.Sirohey, "Human and Machine Recognition of Faces: A Survey", Proc. IEEE, vol.83, pp.705-741, May 1995.

[3] J. Zhang, Y. Yan and M. Lades, "Face Recognition: Eigenfaces, Elastic Matching, and Neural Nets", Proc. IEEE, vol.85, no.9, pp.1423- 1435, 1997.

[4] "The New York Times, www.nytimes.com/2013/04/19/us/fbi-releasesvideo- of-boston-bombingsuspects.html."

[5] "FEI face database," http://www.fei.edu.br/_cet/facedatabase.html.

[6] P. Phillips, H. Moon, P. Rauss, and S. Rizvi, "The FERET evaluation methodology for face recognition algorithms," in IEEE Conference on Computer Vision and Pattern Recognition (CVPR), June 1997, pp. $137-143$.

[7] T. Sim, S. Baker, and M. Bsat, "The CMU pose, illumination, and expression (PIE) database," in IEEE International Conference on Automatic Face and Gesture Recognition, May 2002, pp. 46-51.

[8] M. Kafai, Le An and Bir Bhanu, "Reference Face Graph for Face Recognition", IEEE transactions on information forensics and security, vol. x, no. x, month 2014, pp 1-12.

[9] A. Punnappurath, A. N. Rajagopalan, Sima Taheri, and Rama Chellappa, "Face Recognition Across Non-Uniform Motion Blur, Illumination, and Pose" IEEE transactions on image processing, vol. 24, no. 7 , july 2015 .

[10] H. Hu and G. de Haan, "Adaptive image restoration based on local robust blur estimation," in Proc. 9th Int. Conf. Adv. Concepts Intell. Vis. Syst., 2007, pp. 461-472.

[11] M. Nishiyama, A. Hadid, H. Takeshima, J. Shotton, T. Kozakaya, and O. Yamaguchi, "Facial deblur inference using subspace analysis for recognition of blurred faces," IEEE Trans. Pattern Anal. Mach. Intell., vol. 33, no. 4, pp. 838-845, Apr. 2011.

[12] H. Zhang, J. Yang, Y. Zhang, N. M. Nasrabadi, and T. S. Huang, "Close the loop: Joint blind image restoration and recognition with sparse representation prior," in Proc. Int. Conf. Comput. Vis., Nov. 2011, pp. 770-777.

[13] T. Ahonen, E. Rahtu, V. Ojansivu, and J. Heikkila, "Recognition of blurred faces using local phase quantization," in Proc. 19th Int. Conf. Pattern Recognit., Dec. 2008, pp. 1-4.

[14] R. Gopalan, S. Taheri, P. Turaga, and R. Chellappa, "A blur-robust descriptor with applications to face recognition,” IEEE Trans. Pattern Anal. Mach. Intell., vol. 34, no. 6, pp. 1220-1226, Jun. 2012.

[15] I. Stainvas and N. Intrator, "Blurred face recognition via a hybrid network architecture," in Proc. 15th Int. Conf. Pattern Recognit., vol. 2. Sep. 2000, pp. 805-808.

[16] P. Vageeswaran, K. Mitra, and R. Chellappa, "Blur and illumination robust face recognition via settheoretic characterization,” IEEE Trans. Image Process., vol. 22, no. 4, pp. 1362-1372, Apr. 2013.

[17] K.-C. Lee, J. Ho, and D. Kriegman, "Acquiring linear subspaces for face recognition under variable lighting,” IEEE Trans. Pattern Anal. Mach. Intell., vol. 27, no. 5, pp. 684-698, May 2005.

[18] S. Biswas, G. Aggarwal, and R. Chellappa, "Robust estimation of albedo for illumination-invariant matching and shape recovery," IEEE Trans. Pattern Anal. Mach. Intell., vol. 31, no. 5, pp. 884-899, May 2009.

[19] T. Zhang, Y. Y. Tang, B. Fang, Z. Shazg, and X. Liu, "Face recognition under varying illumination using gradientfaces,” IEEE Trans. Image Process., vol. 18, no. 11, pp. 2599-2606, Nov. 2009.

[20] X. Tan and B. Triggs, "Enhanced local texture feature sets for face recognition under difficult lighting conditions," in Analysis and Modeling of Faces and Gestures (Lecture Notes in Computer Science), vol. 4778. Berlin, Germany: Springer-Verlag, 2007, pp. 168-182.

[21] C. A. Hansen, "Face Recognition", Institute for Computer Science University of Tromso, Norway.

[22] Shang-Hung Lin, "An Introduction to Face Recognition Technology" information science issue on multimedia informing technologies-part-2, vol-3,no.-1,2000.

[23] Divyarajsinh N. Parmar1, Brijesh B. Mehta, "Face Recognition Methods \& Applications" Divyarajsinh N Parmar et al ,Int.J.Computer Technology \& Applications,Vol 4 (1),84-86

[24] Lin, S.-H., Kung, S.Y., and Lin, L.-J., "Face Recognition/Detection by Probabilistic Decision-Based Neural Network", IEEE Trans. Neural Networks, vol. 8, No. 1, pp.114-132, Jan. 1997.

[25] Shengcai Liao, Anil K. Jain and Stan Z. Li, "A Fast and Accurate Unconstrained Face Detector", IEEE transactions on pattern analysis and machine intelligence, Volume: 38, Issue: 2, pp. 211-223, Feb. 2016.

[26] Keyurkumar Patel, Hu Han and Anil K. Jain, "Secure Face Unlock: Spoof Detection on Smartphones" IEEE transactions on information forensics and security, vol. 11, no. 10, october 2016.

[27] Ranbeer Tyagi, GS Tomar, L. Shrivastava, "Unconstrained Face Recognition Quality: A Review", IJSIP, Vol. 9, No.11, pp.199-210, Nov 2016. 